\title{
EVALUATION AND OUTCOME OF DIFFERENT SURGICAL TECHNIQUES FOR POSTINTUBATION TRACHEOESOPHAGEAL FISTULAS
}

Paolo Macchiarini, $\mathrm{MD}, \mathrm{PhD}^{\mathrm{a}}$

Jean-Philippe Verhoye, $\mathrm{MD}^{\mathrm{b}}$

Alain Chapelier, $\mathrm{MD}, \mathrm{PhD}^{\mathrm{b}}$

Elie Fadel, $\mathrm{MD}^{\mathrm{b}}$

Philippe Dartevelle, $\mathrm{MD}^{\mathrm{b}}$
Objective: We evaluated the outcome of different surgical techniques for postintubation tracheoesophageal fistula. Methods: Thirty-two consecutive patients aged $51 \pm 23$ years had tracheoesophageal fistulas resulting from a median of 30 days of mechanical ventilation via endotracheal (n $=12)$ or tracheostomy $(n=20)$ tubes. Tracheoesophageal fistulas were $2.5 \pm 1.2 \mathrm{~cm}$ long and were associated with a tracheal $(n=10)$ or subglottic $(n=3)$ stenosis in 13 patients. Results: All but 3 patients were weaned from respirators before repair. All operations were done through cervical incisions and included direct division and closure $(\mathbf{n}=$ 9), esophageal diversion $(n=3)$, muscle interposition $(n=6)$, or, more recently, tracheal or laryngotracheal resection and anastomosis with primary esophageal closure $(n=14)$. Nine thyrohyoid and two supralaryngeal releases reduced anastomotic tension. Twenty-three patients $(74 \%)$ were extubated after the operation $(n=16)$ or within 24 hours $(n$ $=7$ ), and 7 required a temporary tracheotomy tube. One postoperative death $(3 \%)$ was associated with recurrent tracheoesophageal fistula. Seven complications $(22 \%)$ included recurrent tracheoesophageal fistula $(n=1)$, delayed tracheal stenosis $(n=2)$, dysphagia $(n=2)$, and recurrent nerve palsy $(n=2)$. Complications necessitated reoperation $(n=1)$, dilation $(n=2)$, definitive tracheostomy $(n=1)$, Montgomery $T$ tubes $(n$ $=1)$, and Teflon injection of the vocal cords $(n=1)$. Twenty-nine patients $(93 \%)$ had excellent $(n=24)$ or $\operatorname{good}(n=5)$ anatomic and functional long-term results. Complications have been less common (7\% vs $38 \%$ ) and long-term results better $(93 \%$ vs $65 \%)$ recently with tracheal or laryngotracheal resection and anastomosis with primary esophageal closure as compared with previous procedures. Conclusions: Postintubation tracheoesophageal fistula is usually best treated with tracheal or laryngotracheal resection and anastomosis with primary esophageal closure even in the absence of tracheal damage. ( $J$ Thorac Cardiovasc Surg 2000;119:268-76)
From the Departments of Thoracic and Vascular Surgery, Heidehaus Hospital, Hannover Medical School, Hannover, Germany, ${ }^{a}$ and Thoracic and Vascular Surgery and Heart-Lung Transplantation, Hôpital Marie-Lannelongue, Le Plessis Robinson, Paris-Sud University, France. ${ }^{\mathrm{b}}$

Read at the Twenty-fifth Annual Meeting of The Western Thoracic Surgical Association, Olympic Valley (Lake Tahoe), Calif, June 23-26, 1999.

Received for publication June 29, 1999; revisions requested Sept 10, 1999; revisions received Oct 14, 1999; accepted for publication Oct 19, 1999.

Address for reprints: Paolo Macchiarini, MD, PhD, Department of Thoracic and Vascular Surgery, Heidehaus Hospital (Hannover Medical School), Am Leineufer, 70, 30419 Hannover, Germany (E-mail: pmacchiarini@compuserve.com).

Copyright @ 2000 by Mosby, Inc.

$0022-5223 / 2000 \$ 12.00+0 \quad \mathbf{1 2 / 6 / 1 0 3 7 2 6}$ lthough the widespread use of high-volume lowApressure cuffs has reduced the incidence of cuffrelated tracheoesophageal fistulas (TEFs) to $0.5 \%$ in patients undergoing tracheostomy, ${ }^{1,2}$ long-term intubation still accounts for the majority of acquired, nonmalignant TEFs because of either overinflation of the cuff or placement of small tracheostomy tubes necessitating overinflation of their cuffs to provide airway sealing. ${ }^{3}$ Associated risk factors include excessive motion of the tube, infections, hypotension, steroids, and diabetes. ${ }^{4}$

Operative closure of TEF is necessary because spontaneous closure is so rare..$^{2,3,5}$ Because of the variety of surgical options to repair a TEF, including direct closure of the tracheal and esophageal defects with or without pedicled muscle flaps, ${ }^{6-7}$ tracheal closure with 
Table I. Profile of patient demographics

\begin{tabular}{lc}
\hline Characteristics & Number $(n=32)$ \\
\hline Age (median, range) & 52 y $(19-84 \mathrm{y})$ \\
Sex (male vs female) & $14(43 \%)$ vs $18(57 \%)$ \\
Reason for mechanical ventilation & \\
Medical conditions & $11(34 \%)$ \\
Postoperative complications & $9(27 \%)$ \\
Post-traumatic complications & $11(34 \%)$ \\
Suicide & $1(5 \%)$ \\
Tracheotomy (present vs absent) & $20(63 \%)$ vs $12(37 \%)$ \\
\hline
\end{tabular}

Data are expressed as number (n) of observations.

an esophageal patch, ${ }^{8}$ segmental tracheal resection, and anastomosis with esophageal closure ${ }^{9}$ and esophageal diversion, ${ }^{10}$ the timing and type of repair are still debated. We here evaluated the surgical outcome of postintubation TEF performed before, during, and after the transition from the frequent use of direct or indirect closure or esophageal diversion to the routine use of segmental tracheal resection and anastomosis with esophageal closure.

\section{Patients and methods}

Thirty-two patients with a TEF have been referred to us since January 1981 (Table I). All but 1 of the patients were operated on at Hôpital Marie-Lannelongue. Two patients had prior surgical failures. All but 4 patients were intubated with an orotracheal tube, and the overall median intubation time was 30 days (range, 2-225 days). Twenty (63\%) patients had both a tracheostomy tube and a nasogastric tube in place on arrival. Overall, the diagnosis of TEF was made at a median of 35 days (range, 23-180 days) after the initial intubation, before $(\mathrm{n}=21,66 \%)$, at $(\mathrm{n}=1,3 \%)$, or after $(\mathrm{n}=10,31 \%)$ extubation. The diagnostic delay was significantly $(P<.001)$ longer in patients with medical complications (53 \pm 18 days) than in post-traumatic ( $32 \pm 4$ days) or postoperative (18 \pm 6 days) TEFs.

All patients had a septic syndrome on referral, and 17 (53\%) patients had methicillin-resistant Staphylococcus aureus and blood cultures positive for Streptococcus D, anaerobic, or Candida albicans. Twenty-three (73\%) patients had at least three of the following conditions: body weight loss of $15 \%$ or more while in the intensive care unit, severe hypoproteinemia, electrolyte imbalance, functional or organic kidney insufficiency, and cardiorespiratory failure or coma.

Anatomic patterns of the TEF. The anatomic patterns of the preoperative TEF are outlined in Table II. As shown, the mean length of the TEF was $2.5 \pm 1.2 \mathrm{~cm}$ (range, $1-6 \mathrm{~cm}$ ). Six patients (19\%) had extensive tracheomalacia. The tracheoesophageal tract was stenotic at the subglottic $(\mathrm{n}=3)$ or cervical tracheal $(\mathrm{n}=10)$ level in $13(41 \%)$ patients. Three patients had a double tracheal stenosis, at the level of the tracheostomy and tube cuff. The 20 tracheostomies were infected in 5 patients, inflammatory in 10 , and ischemic in 5 .
Table II. Anatomic characteristics of the TEFs

\begin{tabular}{lc}
\hline Characteristics & Number $(n=32)$ \\
\hline Length of the TEF (cm) & $2.5 \pm 1.2$ \\
Distance from vocal cords (cm) & $3.7 \pm 1.6$ \\
Distance from carina (cm) & $4.7 \pm 1.6$ \\
Laryngotracheal stenosis (present vs absent) & $13(41 \%)$ vs $19(59 \%)$ \\
\hline
\end{tabular}

Data are expressed as number (n) of observations \pm standard deviation.

Management before surgical repair. Diagnosis of the TEF was reconfirmed by removing the tracheostomy tube and visualizing, through the tracheostoma, both the TEF and nasogastric tube, either directly under artificial illumination or indirectly through a flexible bronchoscope. Patients were examined with a flexible bronchoscope to define the site of the TEF, to identify its relationship to the vocal cords, carina, and orifice of the tracheotomy, and to record the presence of circumferential stenosis, inflammation, or injury to the tracheal wall at the cuff level. All patients were preoperatively stabilized with adequate nutritional provision and complete infectious control. ${ }^{1,3,9}$ Removal of the indwelling nasogastric tube was necessary in 26 patients, placement of a new lowpressure high-volume tracheostomy tube whose cuff remained below the site of the fistula in 8 patients, and positioning of draining gastrostomy and feeding jejunostomy tubes in 26 patients.

Anesthesia. The same principles of tracheal surgery were systematically used. To allow spontaneous ventilation during the operation and early extubation, continuous infusion techniques with short-acting muscle relaxants and rapidly metabolized intravenous anesthetic agents and short-acting opioids were used. Distal ventilation was as follows: (1) In patients without a tracheostomy and with a small TEF not requiring tracheal resections, a small orotracheal tube was passed distal to the lesion. (2) In patients with a more extensive TEF, a standard orotracheal tube was placed proximal to the tracheal lesion and small volumes were used. As an alternative, initial ventilation was obtained through the orifice of the tracheostomy with the endotracheal tube pushed distal to the diseased tracheoesophageal segment. Once the airway was opened, all further tracheobronchial intubations were accomplished by sterile tubes inserted into the distal trachea and connected by the anesthesiologist with a sterile connecting tubing. (3) For TEFs located just above the carina, ventilatory assistance was obtained with high-frequency jet ventilation administered through small catheter(s) placed either across or through the stenotic or transected airway into both main bronchi or into the left lung. Alternatively, both lungs were ventilated through two small (No. 5) endotracheal tubes placed in each main bronchus.

Operative technique. Direct or indirect defect closure or esophagocolonoplasty was done at the beginning of our experience, whereas tracheal resection and anastomosis and esophageal closure have been adopted as the routine treatment since 1991 


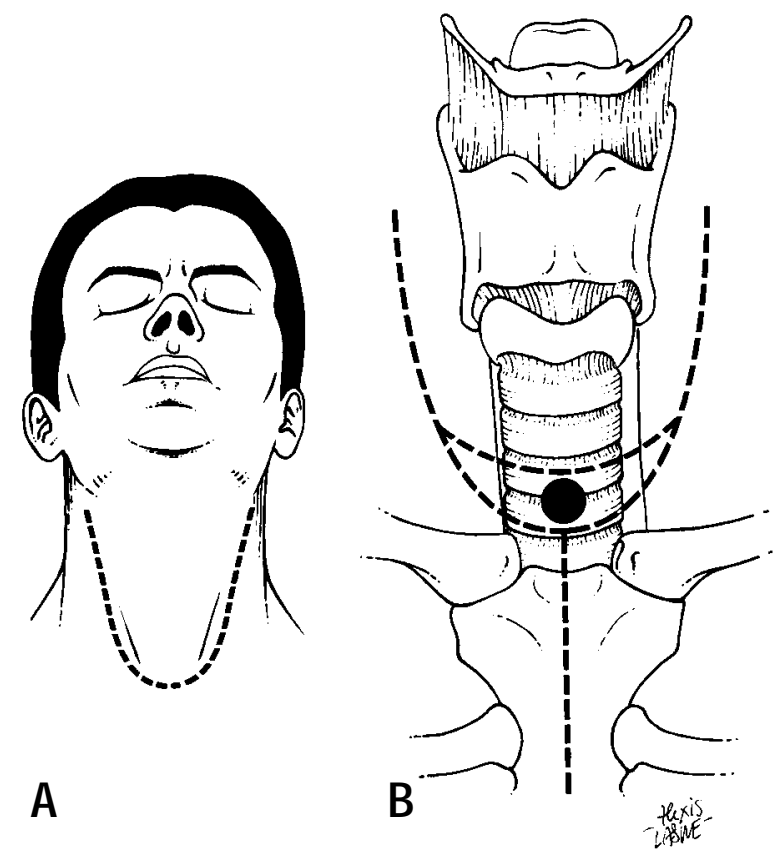

A

Fig 1. A, U-shaped cervical incision. Inferiorly, the incision can be prolonged vertically just below the manubrial notch to include an upper median sternotomy. B, The tracheal stoma can be simply resected while performing the collar incision if it does not directly communicate with the fistula or associate with a tracheal stenosis or injury such that tracheal reconstruction would not be possible. In these last situations the stoma should be left in place and closed later with a myoplasty. If the patient needs postoperative ventilatory support, the tracheal stoma might be left in place. After removal of the endotracheal tube, it usually closes spontaneously. In some cases it fails to close when prolonged intubation has allowed epithelialization between the skin and the tracheal mucosa. In this case, a simple one-stage closure with an inverted skin or myocutaneous flap may produce an epithelialized tracheal wall.

Direct closure of the tracheal and esophageal defects. This technique was used in patients with small TEFs and a morphologically normal trachea not requiring ventilatory support. Exposure was always obtained by an incision in the lateral left presternocleidomastoid muscle. The sternocleidomastoid muscle was dissected from the underlying muscles and the omohyoid and prethyroid muscles were divided to expose the jugular vein, carotid artery, and thyroid gland. The middle thyroid vein, if present, and the inferior thyroid artery were ligated and divided, and the thyroid, pharynx, and larynx were retracted forward and contralaterally, putting the deep cervical fascia under tension. The recurrent laryngeal nerve was then exposed but not dissected in the tracheoesophageal groove and identified at a location remote from the fistula. In most cases, identification of the fistulous tract was not simple and required a more extensive dissection than expected. Once the fistulous tract was dissected and individ-

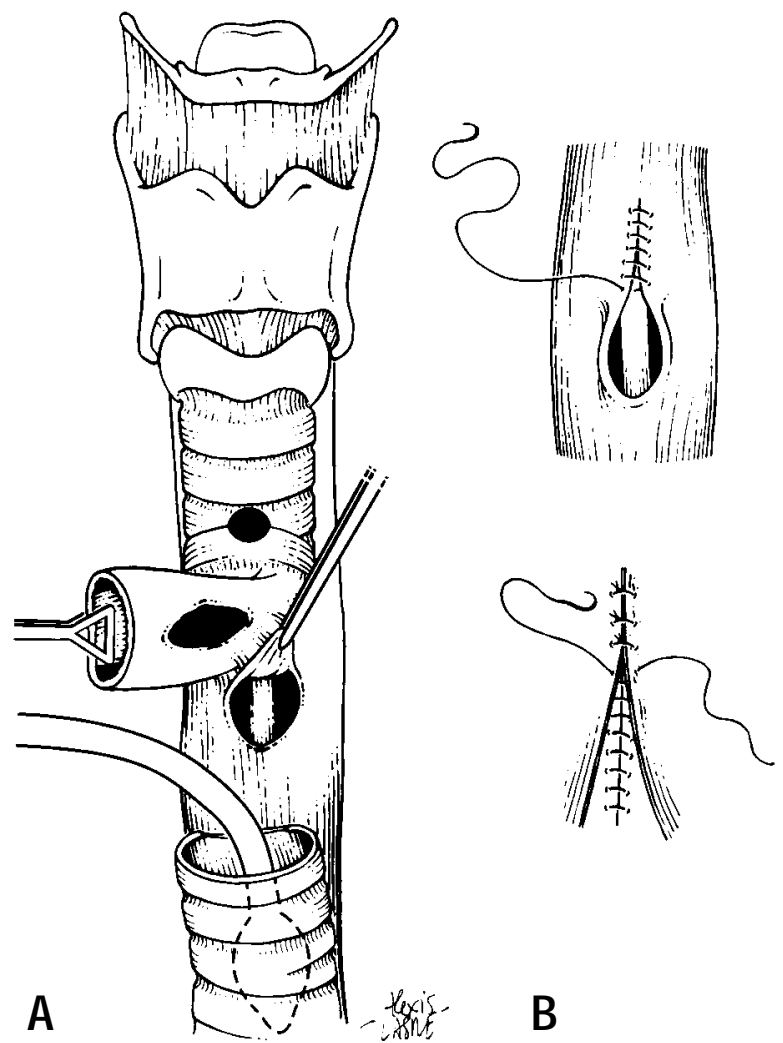

Fig 2. In patients whose trachea is stenotic at the site of the fistula, the trachea is divided below and above the damaged area (including eventually the stoma) and ventilation is obtained by inserting a cross-field endotracheal tube into the distal tracheal airway. The opening of the airway gives complete exposure to the esophageal defect. Mucosal (A) and muscular (B) esophageal wall closure with interrupted sutures is then fashioned over a nasogastric tube.

ualized, it was divided. Closure of the membranous tracheal defect was then accomplished either directly with interrupted sutures of 4-0 polydioxanone (PDS, Ethicon, Inc, Somerville, $\mathrm{NJ}$ ) or indirectly by transposing and suturing surrounding strap muscles (cleidomastoid, sternothyroid, sternohyoid, or deltopectoral muscles) to the tracheal defect with interrupted 3-0 or 4-0 polyglactin (Vicryl, Ethicon) sutures. The esophageal defect was then closed by a 2-layer closure after debridement of the fistulous edges. The inner esophageal mucosal layer was closed followed by closure of the outer esophageal muscle over the mucosal layer with interrupted 30 Vicryl sutures. By way of avoiding close contact of the two suture lines and preventing recurrence, a pedicle flap of strap muscle may be interposed between the esophagus and trachea or the esophagus can be mobilized, rotated, and fixed to the prevertebral plane.

Tracheal resection and anastomosis with primary esophageal closure. These were the minor modifications of the original technique superbly described by Grillo, 
Moncure, and McEnany. ${ }^{9}$ Through an anterior cervical Ushaped incision (Fig 1), including eventually the tracheostomy stoma, if present, the skin, subcutaneous fat, and platysma were elevated as one layer and the flap was raised to the level of the suprahyoid region. The sternohyoid muscles were divided in the midline, and the thyroid isthmus was divided and ligated to expose the anterior surface of the trachea. The trachea was freed circumferentially only above and below the site of the fistula, and care was taken to maintain the dissection as close as possible to the outer tracheal surface to avoid injury to both recurrent laryngeal nerves and to preserve the lateral blood supply to the unresected trachea. The trachea was then divided below and above the damaged area, in healthy tissue, and two lateral traction sutures were placed in the distal tracheal wall. It is of paramount importance to assure that the tracheal cut end has a normal diameter and mucosal appearance. Once the distal airway was divided, ventilation was obtained by inserting either a cross-field endotracheal tube (Fig 2) or one or two catheters for high-frequency jet ventilation into the distal tracheal airway. The original endotracheal tube was withdrawn by the anesthetist, and its tip was secured with silk sutures to be guided back through the glottis on completion of the anastomosis. The opening of the airway gives a complete exposure to the esophageal defect. The edges of the esophageal defect were debrided and a two-layer closure was made over a nasogastric tube.

After closure of the esophageal defect, the trachea was reconstructed via a posterior continuous 4-0 PDS suture and several interrupted 3-0 Vicryl sutures anteriorly. Before completion of the anastomosis, the distal ventilation system was removed and the original endotracheal tube advanced beyond the anastomosis line. Excessive anastomotic tension was avoided by performing laryngeal release. The wound was then filled with sterile saline solution to test the anastomosis. The cervical incision was closed by covering the anastomotic line with the thyroid isthmus and by approaching the linea alba cervicalis. If the anastomosis was located behind the innominate artery, a muscle flap was interposed between the suture line and the artery. Two soft multiholed drain catheters were placed.

The mobility of the vocal cords was then assessed. If the airway was adequate and there was no evidence of bilateral recurrent nerve palsy, the patient was extubated immediately after the operation. Patients whose TEF is located in the subglottic region require special care. They cannot be treated with circumferential resection because this would unavoidably divide both recurrent laryngeal nerves and result in collapse of the airway at the subglottic level. In these cases, the operative technique described by Pearson and associates ${ }^{11}$ offers the great advantage of treating such subglottic lesions without damaging the recurrent nerves while conserving laryngeal function. This technique can be successfully applied for those subglottic laryngotracheal strictures extending up to $1 \mathrm{~cm}$ from the vocal cords. It consists in removing the affected cervical trachea and involved subglottic larynx by completely resecting the anterior cricoid arch, partially resecting the posterior cricoid plate (subperichondrially), and
Table III. Early ( $<30$ days) and late ( $>30$ days) surgical complications

\begin{tabular}{lcc}
\hline Type of surgical complication & Early $(n=32)$ & Late $^{*}(n=31)$ \\
\hline Death & $1(3 \%)$ & - \\
Relapse TEF & - & $1(2 \%)$ \\
Anastomotic partial dehiscence & $2(6 \%)$ & - \\
Occlusion of the & $1(3 \%)$ & - \\
$\quad$ esophagocolonoplasty & & - \\
Wound infection & $2(6 \%)$ & - \\
Dysphagia & $15(47 \%)$ & $2(6 \%)$ \\
Dysphonia & $6(19 \%)$ & $2(6 \%)$ \\
Aspiration & $10(33 \%)$ & - \\
Tracheal stenosis & - & $2(6 \%)$ \\
\end{tabular}

Data are expressed as number (n) of observations \pm standard deviation. *Excludes 1 postoperative death.

reestablishing the laryngotracheal continuity by a cricotracheal (posteriorly) and thyrotracheal (anteriorly) anastomosis.

Follow-up studies. All patients were observed until death or last date of follow-up (June 1, 1999). None of them was lost to follow-up, and efficacy of the operation was evaluated by clinical and investigational examinations in all surviving patients. Results were expressed as excellent (without any sequelae) or good (minor sequelae not affecting quality of life), both anatomically and functionally.

\section{Results}

Three patients underwent an emergency operation because the TEF was diagnosed within 24 hours after weaning and the general condition of the patients were excellent, without signs of malnutrition, pulmonary contamination, or sepsis. The remaining 29 patients had elective operations after a median of 15 days (range, 0-110 days) of medical preparation. Among them, $3(10 \%)$ patients could not be weaned from mechanical ventilation before the operation despite the institution of all principles of initial management.

Surgical characteristics. In all but two operations, distal ventilation was obtained with cross-field intubation. Five left lateral cervicotomies were used at the beginning of our experience, but all the other 27 operations were done with a U-shaped cervical incision only. Operations included direct tracheal and esophageal closure in 9 patients (28\%), indirect closure with myoplasty in $6(19 \%)$, a two-stage esophageal exclusion followed by esophageal reconstruction via a retrosternal esophagocolonoplasty in $3(9 \%)$, and subglottic $(n=4)$ or tracheal $(n=10)$ resection and anastomosis with esophageal closure in 14 patients (44\%). During this last procedure, the mean length of the resected trachea was $4.6 \pm 1.2 \mathrm{~cm} ; 9$ thyrohyoid and 2 supralaryngeal releases reduced anastomotic tension.

Morbidity and mortality. Twenty-three (74\%) 
Table IV. Characteristics of patients having a 2-stage esophageal diversion and esophagocolonoplasty reconstruction

\begin{tabular}{llll}
\hline Characteristics & \multicolumn{1}{c}{ Patient 1 } & \multicolumn{1}{c}{ Patient 2 } & \multicolumn{1}{c}{ Patient 3 } \\
\hline Sex, age (y) & M, 42 & M, 76 & F, 36 \\
Etiology & Necrotic pancreatitis & Respiratory insufficiency & Fibrotic mediastinitis \\
Tracheostomy & Yes & Yes & Yes \\
Feeding & Jejunostomy & Jejunostomy & Jejunostomy \\
Surgical indication & Redo for bipolar esophageal exclusion & Unipolar esophageal exclusion & Unipolar esophageal exclusion \\
Operative technique & Retrosternal esophagocolonoplasty & Retrosternal esophagocolonoplasty & Retrosternal esophagocolonoplasty \\
Early complications & Retrosternal graft occlusion & Bilateral pneumonia and aspiration & Dysphagia \\
Late complications & Diverticulum of the esophageal stump & Definitive tracheostomy & Fibrotic mediastinitis \\
Follow-up & 16 y & Death at 1 y of respiratory & Death at 18 mo of progressive \\
& & insufficiency & mediastinitis \\
\hline
\end{tabular}

patients were extubated immediately after the operation $(n=16)$ or within 24 hours $(n=7)$. Because of the extensive tracheal resection and to decompress the laryngotracheal axes, a tracheostomy tube was left in place in 7 patients.

Early ( $<30$ days) and late ( $>30$ days) morbidity and mortality are listed in Table III. One postoperative death $(3 \%)$ occurred after indirect tracheal (via the left sternocleidomastoid muscle) and two-layer esophageal closure. On the 7 th postoperative day, the patient required mechanical ventilation for bilateral pneumonia with Pseudomonas aeruginosa and Candida albicans culminating in a recurrence of the TEF by day 12 and septic shock thereafter. Seven late complications $(22 \%)$ were successfully managed with reoperation via a tracheal resection and anastomosis $(\mathrm{n}=1)$, tracheal ( $\mathrm{n}$ $=1)$ or esophageal $(n=2)$ dilation, definitive tracheostomy $(\mathrm{n}=1)$, Montgomery $\mathrm{T}$ tubes $(\mathrm{n}=1)$, and unilateral vocal cord Teflon injection $(\mathrm{n}=1)$. Overall, $1(7 \%)$ of 14 patients treated with tracheal resection and anastomosis and direct esophageal closure had a postoperative complication as opposed to 7 (38\%) of the remaining 18 patients receiving other surgical techniques $(P=.036)$. This complication occurred as dysphagia in a patient treated with subglottic and extended $(6 \mathrm{~cm})$ cervical tracheal resection (for a double tracheal stenosis), esophageal closure of a defect crossing the pharyngoesophageal junction, and double laryngeal release.

Long-term anatomic and functional results. All but 2 patients are alive after a median follow-up of 12.5 years. The 2 deaths were related to the underlying disease and occurred 12 and 18 months after the operation as a result of reconstructive esophagocolonoplasty (Table IV). These 2 patients were also the only patients with postoperative bad (major sequelae or definitive tracheostomy) long-term results. The remaining 29 patients $(93 \%)$ had excellent (without any sequelae, $\mathrm{n}=$
24) or good (minor sequelae not affecting quality of life, $\mathrm{n}=5$ ) anatomic and functional long-term results. Excellent anatomic and functional results were more common in patients treated with tracheal resection and anastomosis and esophageal closure $(\mathrm{n}=13,93 \%)$ as opposed to previous operations $(\mathrm{n}=11,65 \%, P=.04)$.

\section{Discussion}

Most TEFs are diagnosed while patients are still receiving assisted ventilation. Since spontaneous closure of TEF is rare, ${ }^{2,3,5,7,12}$ surgical closure should be attempted in each instance. However, repair should be delayed until the patient is in fully stabilized condition and weaned from assisted ventilation. This can be accomplished by following certain principles of initial management. First, the nasogastric tube must be removed because it contributes, along with the tracheostomy tube, to the continuous pressure necrosis of the closely opposed tracheoesophageal walls. Since this mechanism ultimately generates the TEF, it may be speculated that the potential development of the TEF can be avoided if the nasogastric tube is removed early in the intensive care unit. Second, efforts should be specifically directed to avoid stenting or plugging of the tracheoesophageal hole with the endotracheal tube or cuff or endoluminal stent, for instance, by placing a new endotracheal cuff below the fistula. We believe that the long length of the TEF in our series relates to the fact that the majority of our patients had simultaneous nasogastric and tracheal intubation at some time before referral. We are persuaded that, to some extent, fistula extension resulted from continued pressure in this region and led to "giant" TEF necessitating long segmental tracheal resections. Third, gastric decompression should be obtained by a draining gastrostomy to avoid gastroesophageal reflux, as well as a feeding jejunostomy to correct nutritional deficits. These maneuvers are usually sufficient to avoid the devastat- 
ing pulmonary complications of TEF, improve pulmonary function, reduce duration of mechanical ventilator dependency, reverse the inflammatory status of the fistulous tract, allow nearby tissues to become firm enough to hold sutures, and permit adequate nutritional support. 3,9

Outside the defined treatment principles are those TEFs lying near the carina, for which adequate distal ventilation is troublesome, or those in patients whose underlying disease is controlled without pleuropulmonary contamination, sepsis, or malnutrition, in whom the TEF is diagnosed within 24 to 72 hours after extubation. In both circumstances, surgical repair may be attempted, since operations resulted in a major complication in only 1 of 6 patients requiring emergency surgery in our experience.

Once the patient is weaned, the surgical repair can be accomplished by simple division and direct or indirect closure of the fistula, segmental tracheal resection, and primary anastomosis with esophageal closure or esophageal diversion. ${ }^{6-10}$ After having used the simple division and fistula closure or esophageal diversion as the predominant techniques for almost one decade, we began, in 1991, a gradual transition to the routine use of the anterior approach proposed by Grillo, Moncure, and McEnany. ${ }^{9}$ The reasons were both subtle and multiple. There were no data on the superiority of one technique, and all had their pros and cons. The left lateral approach used for the simple division and repair gives a limited and unilateral exposure of the fistulous tract, even after partial upper sternotomy, and requires exposure of the left recurrent laryngeal nerve. Identification of the fistulous tract may not be simple and usually requires a more extensive dissection than expected. The tracheal membranous dehiscence is usually longer and larger than that expected from the preoperative endoscopic evaluation, and there is usually some mediastinal scar around the tracheal split. Hence the separation of the tracheal and esophageal lines requires esophageal rotation and thus esophageal devascularization and can result in late tracheal stenosis at the level of the repair or muscle interposition, as was observed early in our experience. Among the 15 indirect or direct tracheal and esophageal closures, 2 patients with TEF had a relapse and 1 of them died, for a $13 \%$ incidence of relapse and a $7 \%$ mortality rate for this technique.

Diversion of the esophageal contents and partial or total exclusion of the esophagus in continuity with the fistula $^{13}$ is by far the most complicated, risky, and invariably unsuccessful surgical repair of postintubation TEF, ${ }^{1,5-7}$ as it was in our hands as well. The rationales for complete diversion are that the excluded

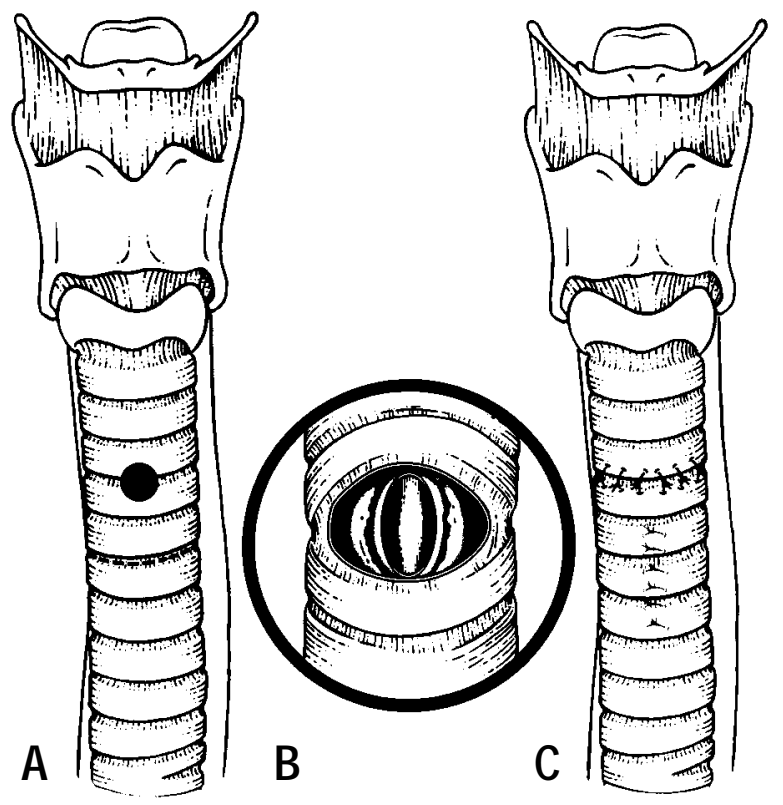

Fig 3. In patients whose trachea is normal, transection of the trachea at the midline level of the fistula (A) gives an anterior view of the entire defect (B). After esophageal closure, the anterior and posterior tracheal defects are closed with continuous and interrupted sutures as described in the text. Note (C) that the esophageal and tracheal anastomotic lines are not at all in contact with each other because of the reduced tracheal but unchanged esophageal length.

esophageal segment (1) withstands elevated mean inspiratory pressures, (2) creates a new and solid posterior tracheal wall, and (3) suppresses a blind esophageal pouch or significant dead space ventilation without compromising delayed tracheal and esophageal reconstructions by a second-stage tracheal resection and primary reconstruction and colon or free jejunal interposition. Esophageal diversion should be used very selectively, that is, if the principles of preoperative management in ventilator-dependent patients fail, because the morbidity and mortality rates after esophageal diversion are remarkably higher than those of the other techniques. This observation was confirmed in our series as well. The only two bad anatomic and functional results we observed occurred in patients having a two-stage esophageal diversion followed by esophagocolonoplasty.

Although these were only clinical observations and not documented by statistical quantitative measurements, the introduction of the anterior approach originally described by Grillo, Moncure, and McEnany ${ }^{9}$ and subsequently updated by Mathisen and associates, ${ }^{1}$ including tracheal resection of the stenotic segment 
(when present), primary tracheal reconstruction, and two-layer esophageal repair, was a pleasant surprise. Because our experience with tracheal surgery was increasing, in the next year we gradually underwent the transition from the frequent use of the previously described techniques to the routine use of the anterior approach, irrespective of the presence of tracheal stenotic damage. As experience increased, it became evident that Grillo's approach displays the following advantages over lateral direct division and closure. The repair is fashioned by an anterior incision that does not require either extensive tracheal or esophageal devascularization but only a single root of dissection. The recurrent laryngeal nerves are less likely to be injured. The operative field has a larger surface and gives an anterior, bilateral, and thus complete exposure of the entire tracheoesophageal damage, including those intrathoracic tracheal and esophageal defects otherwise unreachable from the lateral exposure. Since it treats the associated tracheal defect by a segmental resection, the risks of tracheal complications that may follow the lateral approach are almost canceled. Moreover, the esophageal and tracheal anastomotic lines are not in contact with each other because of the reduced tracheal but unchanged esophageal length (Fig 3). Therefore eventual muscle interpositions or esophageal rotations are unnecessary. This prevents late membranous tracheal wall compression or stenosis at the level of the muscle or soft flap interposition, as we observed after other forms of repairs.

One may question whether the routine transection or resection of the airway and resection of the fistulous tracheal tract is worthwhile. On the basis of our learning curve and presented results, we believe that in experienced hands this simple operative step adds no morbidity but does add significant early and long-term clinical benefit, inasmuch as many patients have some sort of tracheal damage. Moreover, the quality of exposure to the esophageal defect after the tracheal transection and/or resection is, in our experience, by far so superior to any other approach that it enhances the safety and the precise visualization of the entire tracheoesophageal defect(s), permitting at best the most detailed completion of the operation. Unsurprisingly, we had only one complication in a patient whose subglottic TEF was repaired through an extensive $(6 \mathrm{~cm})$ tracheal resection necessitating supralaryngeal release. This approach can also be applied to those patients with end-stage respiratory function willing to sacrifice their larynx and undergo a tracheal diversion. If the esophageal fistula and the trachea are closed above the defect and a definitive tracheostomy is performed, the dead respiratory space is reduced, respiration assured, and the TEF ultimately repaired.

In conclusion, the surgical experience learned over an almost 20-year period with the management of postintubation TEF underscores that surgical repair should be delayed until the patient is stabilized and weaned from ventilation. This can almost always be accomplished by removing the nasogastric tube, if present, placing a new tracheostomy tube whose cuff rests below the fistula, and placing a feeding jejunostomy and draining gastrostomy. These guidelines are usually sufficient to prepare patients for operation. The anterior approach as described by Grillo, Moncure, and McEnany ${ }^{9}$ was routinely applied in the last 14 patients and resulted in no mortality and excellent or good anatomic and functional results in all but one patient. These figures were significantly worse in patients undergoing other types of surgical repairs, suggesting that Grillo's operation ${ }^{9}$ should be considered the optimal surgical technique to curatively treat postintubation TEF.

\section{REFERENCES}

1. Mathisen DJ, Grillo HC, Wain JC, Hilgenberg AD. Management of acquired nonmalignant tracheoesophageal fistula. Ann Thorac Surg 1991;52:759-65.

2. LeBrigand H, Roy B. Fistules trachéo-oesophagiennes après trachéotomies: à propos de 4 observations. Mem Acad Chir 1966; 92:405-10.

3. Dartevelle P, Macchiarini P. Management of acquired tracheoesophageal fistula. Chest Clin North Am 1996;4:819-36.

4. Montgomery WW. Surgery of the trachea. In: Surgery of the upper respiratory system. Vol II. Philadelphia: Lea \& Febiger: 1989. p. 365-443.

5. Couraud L, Bercovici D, Zanotti L, Velly JF, Dubrez J. Treatment of esophago-tracheal fistulae secondary to respiratory intensive care. Ann Chir 1989;43:677-81.

6. Flege JB. Tracheoesophageal fistula caused by cuffed tracheostomy tube. Ann Thorac Surg 1967;166:153-6.

7. Thomas AN. Management of tracheoesophageal fistula caused by cuffed tracheal tubes. Am J Surg 1972;124:181-9.

8. Bartlett RH. A procedure for the management of acquired tracheoesophageal fistula in ventilatory patients. J Thorac Cardiovasc Surg 1976;71:89-95.

9. Grillo HC, Moncure AC, McEnany MT. Repair of inflammatory tracheoesophageal fistula. Ann Thorac Surg 1976;22:112-9.

10. Utley JR, Dillon ML, Todd EP, Griffen WO, Zeok JV. Giant tracheoesophageal fistula. J Thorac Cardiovasc Surg 1978;75:373-7.

11. Pearson FG, Cooper JD, Nelems JM, Van Nostrand AWP. Primary tracheal anastomosis after resection of the cricoid cartilage with preservation of the recurrent laryngeal nerves. J Thorac Cardiovasc Surg 1975;70:805-16.

12. Thomas AN. The diagnosis and treatment of tracheoesophageal fistula caused by cuffed tracheal tubes. J Thorac Cardiovasc Surg 1973;65:612-9.

13. Urschel HC, Razzuk MA, Wood RE, et al. Exclusion and diversion in continuity for traumatic esophageal perforation. Ann Surg 1974;179:587-9. 


\section{Discussion}

Dr Douglas E. Wood (Seattle, Wash). Dr Macchiarini, I sincerely enjoyed your presentation and concise analysis of your 18-year experience with acquired, benign TEF. Your early management relies on two manuscripts reporting successful correction of acquired TEF by Dr Arthur Thomas, one of the founding members of this Association.

You have clearly outlined the principles of management in these patients-prevention of continued airway contamination, treatment of sepsis, avoidance of TEF extension, aggressive nutritional support, and semi-elective repair after mechanical ventilatory support is no longer required. The key management applications of these principles are the placement of the balloon cuff below the fistula, removing the nasogastric tube, avoidance of continued prosthetic pressure on the fistula site, draining gastrostomy, jejunostomy, and antibiotics. These aspects of preoperative preparation are essential to achieving the good results that you and your group have presented.

Dr Macchiarini, you described the evolution in your practice and your satisfaction with the current technique of routine tracheal resection as a critical component of the repair. I agree that the division of the trachea through an anterior approach provides, by far, the best and broadest exposure for correction of the TEF with minimal extent of dissection. This same approach is useful in other difficult tracheoesophageal problems, such as penetrating trauma to the lower part of the neck or thoracic inlet.

I have three criticisms or additions that I would make to your paper. First, the presence of a nasogastric tube, providing a pressure point against the balloon cuff on the common tracheoesophageal wall, is virtually universal in the etiology of these lesions. These lesions should be avoidable if the nasogastric tube is removed early, even if the endotracheal balloon is overinflated. Second, you performed fairly long resections for these benign lesions, requiring a high use of laryngeal release procedures and their expected complications. Since these lesions are benign, mature, and usually have little inflammation at the time of repair, we believe that the required length of tracheal resection is usually trivial, that is, 1 to $3 \mathrm{~cm}$. We have not needed to perform laryngeal releases in any of our patients undergoing this operation. Third, although the tracheal and esophageal suture lines often are not in direct continuity, they are certainly in close proximity and at risk for a recurrent TEF. Since it adds little time and no morbidity to interpose a sternohyoid or sternothyroid muscle flap between the two suture lines, why do you not add this step to separate the suture lines and attempt to minimize the potential for recurrence?

Dr Macchiarini. To address the last question, you are right that interposing a muscle flap is a good option. Unfortunately, in our early experience when we did the indirect closure through an available muscle flap, it was there that we had major problems with late tracheal stenosis. Of 6 patients, 2 had late tracheal stenosis simply because the muscle was pushing against the posterior tracheal wall. Therefore we abandoned this procedure, favoring the anterior approach.
Concerning the extensive tracheal damage that we observed, again you are correct. Early in our experience we had not observed this problem, but now it seems that more patients have more extensive tracheal damage than before, maybe because of the constant overinflation of the tracheostomy cuff.

Dr Wood. I have one additional question. Dr Macchiarini, the principles that you outlined were violated in 6 patients3 patients underwent emergency rather than elective operations, and 3 patients were still requiring mechanical assistance at the time of surgical repair. What were the reasons for not waiting until the patient was in stable condition and no longer required ventilatory support, and what were the outcomes in these patients?

Dr Macchiarini. Concerning the acute situation, these were TEFs that fortunately were diagnosed very early on, within 72 hours at maximum. We treated these 3 patients very recently and had a good outcome because the trachea was viable and the esophageal tissues were not inflammatory or ischemic. Concerning the patients who were unweanable from mechanical ventilation, unfortunately we were unable to delay the operative repair, despite trying to do so, and in these patients the outcome was associated with a higher rate of complications.

Dr John Benfield (Los Angeles, Calif). Dr Macchiarini, I did not hear you say anything about the recurrent laryngeal nerves. Is that because you avoided them and did not dissect them out in the way that Dr Grillo had recommended? Did you encounter any temporary or permanent recurrent laryngeal nerve palsies?

Dr Macchiarini. That is a technical challenge. As compared to the original technique described by Pearson, we did not look specifically at the recurrent nerve during the operation. We simply stayed as close as possible to the tracheal surface. Even at the subglottic region, we did the operations without identifying the nerves, which is almost impossible in these patients because of the scar tissue.

Dr Benfield. Did any of your patients have recurrent laryngeal nerve paresis either temporarily or permanently?

Dr Macchiarini. There was 1 patient with paresis, but the condition was known before the operation.

Dr Benfield. My second question is prompted by the fact that many of your patients must have had respiratory failure before closure of the TEF. Therefore, episodes of respiratory failure in the postoperative period seem almost inevitable. Please tell us about the management of respiratory function in the postoperative period.

Dr Macchiarini. We tried our best to have patients with borderline respiratory function in good shape before the operation. Those who had borderline respiratory function had the major problems, as well. However, by performing a detailed operation we were able to avoid major functional complications. For instance, $80 \%$ of the patients were extubated either immediately or within 24 hours after the operation. Those patients who had temporary tracheostomy or a longer extubation time were managed medically with antibiotics, physiotherapy, and nitric oxide. Concerning those patients who 
had end-stage respiratory failure, we recently adopted an alternative technique. Patients were asked if they were willing to sacrifice their larynx by allowing us to perform a definitive tracheostomy. We were able to successfully manage these patients by reducing the dead respiratory tract.

Dr Steven Guyton (Seattle, Wash). I would echo Dr Wood's comments about strap muscle use, as it is an integral part of Dr Grillo's operation to interpose strap muscle between the suture lines. Particularly with a very extensive TEF, the posterior wall of the trachea could be closed in a longitudinal fashion, sometimes with the use of part of the esophageal wall. With that, it is almost impossible to have the sutures lines not directly opposed, and in this situation some muscle needs to be placed in between. Resection of the trachea really should be limited to the area where there is cartilaginous damage, because the posterior tracheal wall can be reconstructed. With that, it is rare for a laryngeal release to be necessary.

Dr Macchiarini. Our philosophy was not to reconstruct the posterior damaged tracheal wall but rather to resect it completely.

Dr Richard Whyte (Stanford, Calif). I echo the comments and questions of Dr Wood and Dr Guyton regarding the length of the tracheal resection with respect to the length of the fistula.

My question deals with the frequency with which you see this complication. I find this a vanishingly rare complication. I realize that you have seen 31 -odd cases over 20 years. Could you comment on the distribution of these? Were more of these seen earlier, when high-pressure low-compliance devices were used, rather than in the more recent 10 or 15 years when highly compliant cuffs are more typically used?

Dr Macchiarini. It is a conflicting issue. Early in our experience we saw less extensive tracheoesophageal defects, and now we are seeing more defects that are more severe at the tracheal level and not at the esophageal tissue. This may explain why we favored tracheal resection rather than the conservative muscle interposition between the two organs.

\section{THE THORACIC SURGERY FOUNDATION FOR RESEARCH AND EDUCATION}

\section{Don't Forget Your Foundation Gift}

Your Foundation is making a difference in cardiothoracic surgery. This is possible only because of your support. Please consider an annual gift to The Foundation of appreciated stocks, bonds, or mutual funds. You avoid capital gains tax and earn an income tax deduction by donating rather than selling these assets. This may be better for you than a gift of cash.

This is also a good time to review your estate plans.

- Do you know if the IRS will take $75 \%$ to $85 \%$ of your qualified retirement plan?

- Did you know that a gift to your Foundation can reduce this?

- Did you know that you can increase your net income through a gift to The Foundation?

If you have been thinking of making a charitable contribution to TSFRE, this may be the time to consider a planned gift. Often, this type of giving enables an individual to give a larger gift at a cost that is actually lower than if the gift were to be made outright. You may also find that planned giving enables you to meet other personal financial goals while making significant charitable gifts.

You may also give to The Foundation through a revocable instrument, such as a bequest in your will, or through an irrevocable agreement like a charitable lead trust or a charitable remainder trust. You may also give through a life insurance policy or your retirement plan. For more information about your annual gift or a deferred gift, contact Frank Kurtz, TSFRE Executive Director, 312/644-6610; fax: 312/527-6635; E-mail: frank_kurtz@sba.com.

\section{0-2001 Research Award Applications}

Applications for The Thoracic Surgery Foundation's 2000-2001 program of Research Grants, Fellowships, and Career Development Awards will be available in August 2000. To request an application, please contact Lainie Castle at The Foundation office at 312/464-6100, extension 4798. Applications can also be downloaded from The Foundation's home page, www.tsfre.org, after August 1, 2000. 